\title{
A NEW APPROACH FOR THE STOCHASTIC CASH BALANCE PROBLEM WITH FIXED COSTS
}

\author{
Xin Chen \\ Department of Industrial and Enterprise Systems Engineering \\ University of Illinois at Urbana-Champaign \\ Champaign, IL \\ E-mail:xinchen@illinois.edu \\ DaVID Simchi-LeVI \\ Department of Civil and Environmental Engineering, and \\ The Engineering System Division \\ Massachusetts Institute of Technology \\ Cambridge, MA 02139 USA \\ E-mail:dslevi@mit.edu
}

\begin{abstract}
The stochastic cash balance problem is a periodic review inventory problem faced by a firm in which the customer demands might be positive or negative. At the beginning of each time period, the firm may decide to replenish the inventory or return excess stock. Both the ordering cost and the return cost include a fixed component and a variable component. A holding or penalty cost is charged depending on whether the inventory level is positive or negative. The objective of the firm is to find an ordering and return policy so as to minimize the total expected cost over the entire planning horizon. We show how the concept of symmetric $K$-convexity introduced by Chen and Simchi-Levi $[2,3]$ and the concept of $(K, Q)$-convexity introduced by Ye and Duenyas [13] can be used to characterize the optimal policy for this problem.
\end{abstract}

\section{INTRODUCTION}

The stochastic cash balance problem is a cost minimization problem faced by a firm that has to decide how much cash to hold in order to meet its transaction requirements for a given planning horizon with multiple time periods. At the beginning of each time period, the firm makes decisions about the right amount of cash to hold. In particular, 
the firm might choose to increase or decrease the cash levels. Either transaction can incur a cost, which might include both a fixed component independent of the transaction amount and a variable component that is proportional to the transaction amount. During the time period, a stochastic inflow or outflow of cash is incurred to satisfy the firm's transaction. Therefore, the cash levels of the firm might increase or decrease depending on its net expenses. At the end of the transaction period, the cash levels may be positive or negative depending on whether cash is held or owed. A holding cost is charged when the cash level is positive and a penalty cost is charged if the cash level is negative. The objective of the firm is to find a policy for holding cash so as to minimize the total expected (possibly discounted) cost over the entire planning horizon.

Even though coined with the name cash balance, the stochastic cash balance problem can be used to model several other important applications not related to cash. For instance, it models stochastic inventory management for rented equipment studied by Whisler [12]. It is also closely related to a stochastic inventory control problem with returns (see Feinberg and Lewis [5]) or, more broadly, to reverse logistics (see Fleischmann, Bloemhof-Ruwaarda, Dekker, van der Laana, et al. [7]). Indeed, the stochastic cash balance problem can be regarded as a special type of inventory control problem. Hence, in the following, we will use the term inventory level instead of cash level. We will also use the terms order or return to indicate the increase or decrease of the cash levels.

The stochastic cash balance problem received considerable amount of attention in the 1960s. In particular, Eppen and Fama [4] and Whisler [12] studied this problem assuming that there is no fixed transaction cost for either orders or returns. In this case, they were able to characterize the optimal policy. Girgis [8] studied this problem assuming that there is at most one fixed transaction cost; that is, there is a fixed cost for orders but not for returns, or there is a fixed cost for returns but not for orders. In this case, Girgis showed that the concept if-convexity introduced by Scarf [10] can be used to provide a characterization of the structure of the optimal policy.

When there are fixed costs for both orders and returns, the problem becomes much more difficult. Indeed, Neave [9] showed that the $K$-convexity concept might not be suitable when there are fixed costs for both orders and returns. Nonetheless, Neave partially characterized the structure of the optimal policy of the stochastic cash balance problem by constructing the so-called convex-envelope functions associated with the cost-to-go functions.

In this article, we describe some new insights about the stochastic cash balance problem with fixed costs for both orders and returns. In particular, we are able to utilize the newly developed concept of symmetric $K$-convexity introduced by Chen and Simchi-Levi $[2,3]$ and the concept of $(K, Q)$-convexity (which reduces to symmetric $K$-convexity when $K=Q$ ) introduced by Ye and Duenyas [13] to provide a characterization of the structure of the optimal policy. Compared with Neave [9], our approach is more straightforward and conceptually much simpler. Furthermore, some refined results are available based on this approach. Finally, Feinberg and Lewis [6] extended the structural results to the infinite horizon stochastic cash balance problem. 
The organization of this article is as follows. In Section 2 we introduce the formal model of the stochastic cash balance problem and review some related literature on this model and on the concept of $(K, Q)$-convexity. In Section 3 we show how the concept of $(K, Q)$-convexity and the concept of symmetric $K$-convexity can be used for the stochastic cash balance problem. Finally, we provide some concluding remarks in Section 4.

\section{MODEL}

Consider a firm that has to make ordering or return decisions over a finite planning horizon with a total of $N$ time periods. For convenience, we index periods from 1 to $N$, where 1 is the last period and $\mathrm{N}$ is the first period of the planning horizon.

At the beginning of each time period, an ordering or return decision is made. Let $x$ be the inventory level at the beginning of time period $n$ before a decision is made and let $y$ be the inventory level at the beginning of time period $n$ after an ordering or return decision was made. Lead time for the ordering or return transaction is assumed to be zero and the transaction cost is denoted by $c(y, x)$, which is calculated as follows:

$$
c(y, x)= \begin{cases}K+k(y-x) & \text { if } y>x \\ 0 & \text { if } y=x \\ Q+q(x-y) & \text { if } y<x\end{cases}
$$

where $K \geq 0, Q \geq 0$, and $k+q \geq 0$. Notice that the assumption that $k+q \geq 0$ implies that the unit refund is no more than the unit ordering cost.

Demand in time period $n$, denoted by $\xi_{n}$, is stochastic and can be positive or negative. Negative demand implies that the return from end customers is higher than their purchase. Furthermore, demands in different periods are independent of each other. At the end of time period $n$, inventory holding or penalty cost $l_{n}(z)$ is charged and is a function of $z$, the inventory level at the end of that period. Therefore, the expected inventory holding or penalty cost at period $n$ is given by

$$
L_{n}(y)=E\left\{l_{n}\left(y-\xi_{n}\right)\right\},
$$

where $y$ is the inventory level at the beginning of time period $n$ after an ordering or return decision was made. For technical reasons, we assume the following.

AsSUMPTION 1: The function $L_{n}$ is convex and $\lim _{|z| \rightarrow \infty} L_{n}(z)=\infty$. In addition, there exists finite numbers $x_{n} \leq y_{n} \leq w_{n} \leq z_{n}$ such that

$$
\left(L_{n}\left(y_{n}\right)-L_{n}\left(x_{n}\right)\right) /\left(y_{n}-x_{n}\right)<-k \text { and }\left(L_{n}\left(z_{n}\right)-L_{n}\left(w_{n}\right)\right) /\left(z_{n}-w_{n}\right)>q \text {. }
$$

Those technical assumptions are imposed to avoid trivial complications and are the same as those imposed by Neave [9]. If these assumptions are violated, the first 
inequality implies that it is never optimal to order and the second inequality implies that it is never optimal to return.

The objective of the firm is to find an ordering and return policy so as to minimize the total expected (possibly discounted) ordering or return costs plus inventory holding or penalty costs over the entire planning horizon. Similarly to classic stochastic inventory control problems, the stochastic cash balance problem can be formulated and analyzed by a dynamic program.

Let $C_{n}(x)$ be the cost-to-go function starting at the beginning of a period when there are $n$ periods left in the planning horizon and the initial inventory level is $x$. The dynamic programming recursion is

$$
C_{n}(x)=\min _{y}\left\{c(y, x)+L_{n}(y)+\gamma E\left[C_{n-1}\left(y-\xi_{n}\right)\right]\right\},
$$

where $\gamma \in(0,1]$ is a discount factor. For simplicity of presentation, we assume that $C_{0}(x)=0$. Even though this assumption seems to be arbitrary, we note that this assumption can be easily extended.

Define $H_{n}(x)=L_{n}(x)+\gamma E\left\{C_{n-1}\left(x-\xi_{n}\right)\right\}$. It is not hard to see that with Assumption $1, H_{n}(x) \rightarrow \infty$ and, thus, $C_{n}(x) \rightarrow \infty$ for $|x| \rightarrow \infty$. In addition, under mild conditions on the random variable $\xi_{n}$ and the inventory holding and penalty cost function $l_{n}(x),{ }^{1}$ one can show by induction that $C_{n}(x)$ is continuous. Indeed, since

$$
C_{n}(x)=\min \left\{H_{n}(x), \min _{y \geq x}\left\{K+k(y-x)+H_{n}(y)\right\}, \min _{y \leq x}\left\{Q+q(x-y)+H_{n}(y)\right\},,\right.
$$

$C_{n}(x)$ is continuous as long as $C_{n-1}(x)$ is since the minimum and expectation operators preserve continuity provided that the mild conditions on $\xi_{n}$ and $l_{n}(x)$ hold. Thus, throughout this article, we assume that $C_{n}(x)$ is continuous. With this assumption, the minimum in the dynamic program (1) can be attained. Specifically, we will use $y_{n}(x)$ to denote an optimal solution for problem (1).

Eppen and Fama [4] and Whisler [12] studied a special case of the stochastic cash balance problem. In particular, they assumed that $K=Q=0$. Under this assumption, they showed that at time period $n$, there exist two parameters $T_{n}$ and $U_{n}$ with $T_{n} \leq U_{n}$, such that the optimal inventory level $y_{n}(x)$ at the beginning of time period $n$ after a decision is made satisfies

$$
y_{n}(x)= \begin{cases}T_{n} & \text { if } x \leq T_{n} \\ x & \text { if } x \in\left(T_{n}, U_{n}\right) \\ U_{n} & \text { if } x \geq U_{n}\end{cases}
$$

Girgis [8] studied another special case of the stochastic cash balance problem. The author assumed that $K>0, Q=0$, and $k q>0$ or $K=0, Q>0$, and $k q>0$. Girgis showed that when $K>0, Q=0$, and $k q>0$, the cost-to-go, $C_{n}(x)$, is $K$-convex, and as a consequence, there exist three parameters $t_{n}, T_{n}$, and $U_{n}$ with $t_{n} \leq T_{n} \leq U_{n}$ such that the optimal inventory level $y_{n}(x)$ at the beginning of time period $n$ after a decision 
is made has the form

$$
y_{n}(x)= \begin{cases}T_{n} & \text { if } x \leq t_{n} \\ \in\left[t_{n}, x\right] & \text { if } x \in\left(t_{n}, U_{n}\right) \\ U_{n} & \text { if } x \geq U_{n}\end{cases}
$$

By symmetry, if $K=0, Q>0$, and $k q>0$, there exists three parameter $T_{n} \leq U_{n} \leq u_{n}$ such that the optimal policy has the structure

$$
y_{n}(x)= \begin{cases}T_{n} & \text { if } x \leq T_{n} \\ \in\left[x, u_{n}\right] & \text { if } x \in\left(T_{n}, u_{n}\right) \\ U_{n} & \text { if } x \geq u_{n} .\end{cases}
$$

Neave [9] studied the stochastic cash balance problem with general ordering and return costs (i.e., when $K, Q>0$ and $k, q \geq 0$ ). He provided an example that shows that a simple policy (analogous to a two-sided $(s, S)$ policy) is not necessarily optimal and the concept $K$-convexity might not be appropriate even when $K=Q>0$, and $k=q=0$. He developed a technique that constructs convex upper and lower bounds (so-called convex envelope functions) on the nonconvex cost-to-go functions $C_{n}(x)$ to provide a characterization of the optimal policy.

Neave [9] showed that at time period $n$, there exist six parameters $t_{n}, t_{n}^{+}, T_{n}, U_{n}$, $u_{n}^{-}$, and $u_{n}$ with $t_{n} \leq t_{n}^{+} \leq T_{n} \leq U_{n} \leq u_{n}^{-} \leq u_{n}$, such that the optimal inventory level $y_{n}(x)$ at the beginning of time period $n$ after a decision is made satisfies

$$
y_{n}(x)= \begin{cases}T_{n} & \text { if } x \leq t_{n} \\ \in\left\{x, T_{n}\right\} & \text { if } x \in\left(t_{n}, t_{n}^{+}\right) \\ \in\left[x, u_{n}^{-}\right] & \text {if } x \in\left[t_{n}^{+}, u_{n}^{-}\right], 0<K<Q \\ x & \text { if } x \in\left[t_{n}^{+}, u_{n}^{-}\right], K=Q>0 \\ \in\left[t_{n}^{+}, x\right] & \text { if } x \in\left[t_{n}^{+}, u_{n}^{-}\right], K>Q>0 \\ \in\left\{x, U_{n}\right\} & \text { if } x \in\left(u_{n}^{-}, u_{n}\right) \\ U_{n} & \text { if } x \geq U_{n} .\end{cases}
$$

In fact, Neave [9] provided a detailed proof for the case $K=Q>0$ and claimed that the same approach can be used to obtain the characterization of the optimal policy when $K \neq Q$. Unfortunately, it is not clear to us how this argument can get through in the case $x \in\left(t_{n}, t_{n}^{+}\right)$when $K>Q>0$ or in the case $x \in\left(u_{n}^{-}, u_{n}\right)$ when $Q>K>0$. This problem has been also independently pointed out by Ye and Duenyas [13].

In this article, we develop some new insights on the stochastic cash balance problem. It was first proven in Chen [1] that the cost-to-go function $C_{n}(x)$ is symmetric $\max \{K, Q\}$-convex, which allows one to provide a characterization of the optimal policy. Building on the concepts of $K$-convexity and symmetric $K$-convexity, Ye and Duenyas [13] introduced the concept of $(K, Q)$-convexity and applied it to analyze 
a capacity investment problem with fixed capacity adjustment costs. They noticed that their capacity investment problem has some similarities to the stochastic cash balance problem. Indeed, in this article we prove that the cost-to-go function $C_{n}(x)$ is in fact $(K, Q)$-convex and the structure of the optimal policy follows directly from this property. In the special case with $K=Q$, the cost-to-go function $C_{n}(x)$ is symmetric $K$ convex. Compared with Neave [9], our approach is straightforward and conceptually simpler. For instance, we do not need to construct upper and lower bounds on the costto-go function, the tool used in Neave's approach. In addition, our approach provides additional insights on the structure of the optimal policy.

In the following, we present the $(K, Q)$-convexity introduced by $\mathrm{Ye}$ and Duenyas [13].

DeFinition 2.1: A real-valued function $f$ is called $(K, Q)$-convex for $K, Q \geq 0$, if for any $x_{0}, x_{1}$ with $x_{0} \leq x_{1}$ and $\lambda \in[0,1]$,

$$
\begin{aligned}
& f\left((1-\lambda) x_{0}+\lambda x_{1}\right) \leq(1-\lambda) f\left(x_{0}\right)+\lambda f\left(x_{1}\right) \\
& \quad+\lambda K+(1-\lambda) Q-\min \{\lambda, 1-\lambda\} \min \{K, Q\} .
\end{aligned}
$$

A function $f$ is called $(K, Q)$-concave if $-f$ is $(K, Q)$-convex.

Notice that the $(K, 0)$-convexity is exactly the $K$-convexity introduced by Scarf [10] for the classical stochastic inventory control problem. Moreover, $(K, K)$ convexity is symmetric $K$-convexity, a concept introduced and applied in Chen and Simchi-Levi $[2,3]$ to analyze a joint inventory control and pricing problem with fixed ordering costs and general demand distributions.

Below we list some properties of the $(K, Q)$-convexity, which are parallel to those properties of the symmetric $K$-convexity in Chen and Simchi-Levi [2,3].

\section{LEMMA 1:}

(a) $A(K, Q)$-convex function is also $\left(K^{\prime}, Q^{\prime}\right)$-convex for $K \leq K^{\prime}$ and $Q \leq Q^{\prime}$. A real-valued convex function is $(0,0)$-convex and hence $(K, Q)$-convex for all $K, Q \geq 0$.

(b) If $g_{1}(y)$ and $g_{2}(y)$ are $\left(K_{1}, Q_{1}\right)$-convex, and $\left(K_{2}, Q_{2}\right)$-convex, respectively, and $\left(K_{1}-Q_{1}\right)\left(K_{2}-Q_{2}\right) \geq 0$, then for $\alpha, \beta \geq 0, \alpha g_{1}(y)+\beta g_{2}(y)$ is $\left(\alpha K_{1}+\right.$ $\left.\beta K_{2}, \alpha Q_{1}+\beta Q_{2}\right)$-convex.

(c) If $g(y)$ is $(K, Q)$-convex and $w$ is a random variable, then $E\{g(y-w)\}$ is also $(K, Q)$-convex, provided $E\{|g(y-w)|\}<\infty$ for all $y$.

(d) Assume that $g$ is a continuous $(K, Q)$-convex function with $K \geq Q$ and $g(y) \rightarrow \infty$ as $|y| \rightarrow \infty$. Define

$$
\begin{gathered}
S \in \operatorname{argmin}_{x}\{g(x)\}, \\
s=\min \{x \mid g(x)=g(S)+K\}, \\
s^{\prime}=\min \{x \mid g(x)=g(S)+K-Q\},
\end{gathered}
$$


and

$$
u=\max \{x \mid g(x)=g(S)+Q\} .
$$

Then $s \leq s^{\prime} \leq S \leq u$ and we have the following results:

(i) $g(s)=g(S)+K$ and $g(y) \geq g(s)$ for all $y \leq s$.

(ii) $g(u)=g(S)+Q$ and $g(y) \geq g(u)$ for all $y \geq u$.

(iii) $g(y) \leq g(z)+Q$ for all $y$ and $z$ with $z \leq y \leq s^{\prime}$.

(iv) $g(y) \leq g(z)+K$ for all $y$ and $z$ with $s^{\prime} \leq y \leq z$.

(v) $g(y) \leq g(z)+K$ for all $y$ and $z$ with $(s+S) / 2 \leq y \leq z$.

PROOF: We only prove part (d)(v). The readers are referred to Ye and Duenyas [13] for the proof of the remaining parts.

Part (d)(iv) implies that it suffices to prove that $g(x) \leq g(S)+K$ for $S \geq x \geq(s+$ $S) / 2$. In fact, let $x=(1-\lambda) s+\lambda S$ with $\lambda \geq 1 / 2$. Then from the $(K, Q)$-convexity of the function $g$, we have that

$$
\begin{aligned}
g(x) & \leq(1-\lambda) g(s)+\lambda g(S)+\lambda K+(1-\lambda) Q-\min \{\lambda, 1-\lambda\} \min \{K, Q\} \\
& =(1-\lambda)(g(s)+K)+\lambda g(S)+\lambda K+(1-\lambda) Q-\lambda Q \\
& =g(S)+K+(1-2 \lambda) Q \\
& \leq g(S)+K .
\end{aligned}
$$

Thus, part (d)(v) holds.

Of course, a result parallel to Lemma 1(d) can be shown for the case when $K \leq Q$.

Later we will see that Lemma 1(d) allows us to provide a characterization of the structure of the optimal policy of the stochastic cash balance problem. Indeed, if $g(x)$ is given by the function $k x+H_{n}(x)$, then Lemma 1(d)(i) implies that $S$ and $s$ give the order-up-to level and the reorder point, respectively, and Lemma 1(d)(iv) and (d)(v) implies that it is optimal not to place an order for an inventory level higher than $\min \left\{s^{\prime},(s+S) / 2\right\}$. On the other hand, if $g(x)$ is given by the function $-q x+H_{n}(x)$, then Lemma 1(d)(ii) implies that $S$ and $u$ give the return-down-to level and the return point, respectively, and Lemma 1(d)(iii) implies that it is optimal not to return for an inventory level below $s^{\prime}{ }^{2}$ We refer to Section 3 for more details.

The following property will be very useful when we analyze the stochastic cash balance problem in the next section.

LEMma $2^{3}:$ Consider a continuous function $f: \Re \rightarrow \Re$ with $\lim _{|x| \rightarrow \infty} f(x)=\infty$. If $f$ is $(K, Q)$-convex, then the function

$$
g(x)=\min _{y \leq x}\{Q \delta(x-y)+f(y)\}
$$

is also $(K, Q)$-convex, where $\delta(x)=1$ for $x>0$ and $\delta(x)=0$ otherwise. Similarly,

$$
h(x)=\min _{y \geq x}\{K \delta(y-x)+f(y)\}
$$

is also $(K, Q)$-convex. 
PROOF: First observe that the assumptions on $f$ guarantee the attainment of the minimum in the definition of $g$ and $h$.

Let $R=\{x \mid g(x)<f(x)\}$ and $E=\{x \mid g(x)=f(x)\}$. Fix $x_{0}, x_{1} \in \mathfrak{R}$ and assume $x_{0} \leq x_{1}$. Define $x_{\lambda}=(1-\lambda) x_{0}+\lambda x_{1}$ for $\lambda \in[0,1]$.

We distinguish four cases.

(1) $x_{0} \in E, x_{1} \in E$. Thus, $g\left(x_{0}\right)=f\left(x_{0}\right)$ and $g\left(x_{1}\right)=f\left(x_{1}\right)$. Note that $g(x) \leq f(x)$ for any $x$ and, in particular, $g\left(x_{\lambda}\right) \leq f\left(x_{\lambda}\right)$. Hence, inequality (2) in the definition of $(K, Q)$-convexity holds (with $f$ replaced by $g$ ).

(2) $x_{0} \in R, x_{1} \in R$. In this case, let $g\left(x_{i}\right)=Q+f\left(y_{i}\right)(i=0,1)$ with $x_{i} \geq y_{i}$; that is, $y_{i}$ achieves the minimum in the definition of $\mathrm{g}$ at $x_{j}$. Define $y_{\lambda}=(1-\lambda)$ $y_{0}+\lambda x_{1}$. It is straightforward to see that $y_{\lambda} \leq x_{\lambda}$ and $g\left(x_{\lambda}\right) \leq Q+f\left(y_{\lambda}\right)$. Therefore,

$$
\begin{aligned}
g\left(x_{\lambda}\right) \leq & Q+f\left(y_{\lambda}\right) \\
\leq & (1-\lambda)\left(Q+f\left(y_{0}\right)\right)+\lambda\left(Q+f\left(x_{1}\right)\right)+\lambda K+(1-\lambda) Q \\
& -\min \{\lambda, 1-\lambda\} \min \{K, Q\} \\
= & (1-\lambda) g\left(x_{0}\right)+\lambda g\left(x_{1}\right)+\lambda K+(1-\lambda) Q-\min \{\lambda, 1-\lambda\} \min \{K, Q\} .
\end{aligned}
$$

(3) $x_{0} \in E, x_{1} \in R$. Let $y_{1}<x_{1}$ be such that $g\left(x_{1}\right)=Q+f\left(y_{1}\right)$. From the definition of the function $g$, we have $f\left(y_{1}\right) \leq f\left(x_{0}\right)$.

We distinguish three subcases.

(a) $x_{\lambda} \geq y_{1}$. In this case,

$$
\begin{aligned}
g\left(x_{\lambda}\right) \leq & Q+f\left(y_{1}\right) \\
= & g\left(x_{1}\right)+(1-\lambda)\left(g\left(x_{0}\right)-f\left(x_{0}\right)\right) \\
\leq & (1-\lambda) g\left(x_{0}\right)+\lambda g\left(x_{1}\right)+(1-\lambda)\left(Q+f\left(y_{1}\right)-f\left(x_{0}\right)\right) \\
\leq & (1-\lambda) g\left(x_{0}\right)+\lambda g\left(x_{1}\right)+(1-\lambda) Q \\
\leq & (1-\lambda) g\left(x_{0}\right)+\lambda g\left(x_{1}\right)+\lambda K+(1-\lambda) Q \\
& -\min \{\lambda, 1-\lambda\} \min \{K, Q\},
\end{aligned}
$$

where the first inequality follows from the definition of the function $g$, the second inequality holds since $x_{1} \in E$ and $x_{1} \in R$, the third inequality holds since $f\left(y_{1}\right) \leq f\left(x_{0}\right)$, and the last inequality holds since $\lambda K \geq$ $\min \{\lambda, 1-\lambda\} \min \{K, Q\}$

(b) $x_{\lambda} \leq y_{1}$ and $f\left(y_{1}\right)-f\left(x_{0}\right) \leq Q-K$. Let $\lambda \leq \mu$ be such that $x_{\lambda}=$ $(1-\mu) x_{0}+\mu y_{1}$.

$$
\begin{aligned}
g\left(x_{\lambda}\right) \leq & f\left(x_{\lambda}\right) \\
\leq & (1-\mu) f\left(x_{0}\right)+\mu f\left(y_{1}\right)+\mu K+(1-\mu) Q \\
& \quad-\min \{\mu, 1-\mu\} \min \{K, Q\}
\end{aligned}
$$




$$
\begin{aligned}
= & (1-\lambda) g\left(x_{0}\right)+\lambda g\left(x_{1}\right)+\lambda K+(1-\lambda) Q \\
& -\min \{\lambda, 1-\lambda\} \min \{K, Q\}+(\mu-\lambda)\left(f\left(y_{1}\right)-f\left(x_{0}\right)\right) \\
& -\lambda Q+(\mu-\lambda)(K-Q)+\min \{\lambda, 1-\lambda\} \min \{K, Q\} \\
& -\min \{\mu, 1-\mu\} \min \{K, Q\} \\
\leq & (1-\lambda) g\left(x_{0}\right)+\lambda g\left(x_{1}\right)+\lambda K+(1-\lambda) Q \\
& -\min \{\lambda, 1-\lambda\} \min \{K, Q\}-\lambda Q+\min \{\lambda, 1-\lambda\} \min \{K, Q\} \\
& -\min \{\mu, 1-\mu\} \min \{K, Q\} \\
\leq & (1-\lambda) g\left(x_{0}\right)+\lambda g\left(x_{1}\right)+\lambda K+(1-\lambda) Q \\
& -\min \{\lambda, 1-\lambda\} \min \{K, Q\}
\end{aligned}
$$

where the first inequality follows from the definition of the function $g$ the second inequality follows from the $(K, Q)$-convexity of the function $f$, the first equality follows from rearranging terms, the third inequality follows from the the assumption that $f\left(y_{1}\right)-f\left(x_{0}\right) \leq Q-K$, and the last inequality is trivial.

(c) $x_{\lambda} \leq y_{1}$ and $f\left(y_{1}\right)-f\left(x_{0}\right) \geq Q-K$. In this case,

$$
\begin{aligned}
g\left(x_{\lambda}\right) \leq & Q+f\left(x_{0}\right) \\
= & (1-\lambda) g\left(x_{0}\right)+\lambda g\left(x_{1}\right)+(1-\lambda) Q+\lambda\left(f\left(x_{0}\right)-f\left(y_{1}\right)\right) \\
\leq & (1-\lambda) g\left(x_{0}\right)+\lambda g\left(x_{1}\right)+(1-\lambda) Q+\lambda(K-Q) \\
= & (1-\lambda) g\left(x_{0}\right)+\lambda g\left(x_{1}\right)+\lambda K+(1-\lambda) Q-\lambda Q \\
\leq & (1-\lambda) g\left(x_{0}\right)+\lambda g\left(x_{1}\right)+\lambda K+(1-\lambda) Q \\
& -\min \{\lambda, 1-\lambda\} \min \{K, Q\},
\end{aligned}
$$

where the first inequality follows from the definition of the function $g$, the first equality holds since $x_{0} \in E$ and $x_{1} \in R$, the second inequality follows from the assumption that $f\left(y_{1}\right)-f\left(x_{0}\right) \geq Q-K$, and the last inequality is trivial.

(4) $x_{0} \in R, x_{1} \in E$. Let $g\left(x_{0}\right)=Q+f\left(y_{0}\right)$ for some $y_{0}<x_{0}$. We distinguish between two cases.

(a) $f\left(y_{0}\right)-f\left(x_{1}\right) \leq K-Q$.

$$
\begin{aligned}
g\left(x_{\lambda}\right) \leq & Q+f\left(x_{0}\right) \\
= & (1-\lambda) g\left(x_{0}\right)+\lambda g\left(x_{1}\right)+\lambda\left(Q+f\left(y_{0}\right)-f\left(x_{1}\right)\right) \\
\leq & (1-\lambda) g\left(x_{0}\right)+\lambda g\left(x_{1}\right)+\lambda K \\
\leq & (1-\lambda) g\left(x_{0}\right)+\lambda g\left(x_{1}\right)+\lambda K+(1-\lambda) Q \\
& -\min \{\lambda, 1-\lambda\} \min \{K, Q\},
\end{aligned}
$$


where the first inequality follows from the definition of the function $g$, the equality follows from the assumption that $x_{0} \in R$ and $x_{1} \in E$, the second inequality follows from the assumption that $f\left(y_{0}\right)-f\left(x_{1}\right) \leq K-Q$, and the last inequality is trivial,

(b) $f\left(y_{0}\right)-f\left(x_{1}\right) \geq K-Q$. Let $\mu \geq \lambda$ be such that $x_{\lambda}=(1-\mu) y_{0}+\mu x_{1}$. Then

$$
\begin{aligned}
g\left(x_{\lambda}\right) \leq & f\left(x_{\lambda}\right) \\
\leq & (1-\mu) f\left(y_{0}\right)+\mu f\left(x_{1}\right)+\mu K+(1-\mu) Q \\
& -\min \{\mu, 1-\mu\} \min \{K, Q\} \\
= & (1-\lambda) g\left(x_{0}\right)+\lambda g\left(x_{1}\right)+\lambda K+(1-\lambda) Q \\
& -\min \{\lambda, 1-\lambda\} \min \{K, Q\}+(\mu-\lambda)\left(f\left(x_{1}\right)-f\left(y_{0}\right)\right) \\
& -(1-\lambda) Q+(\mu-\lambda)(K-Q)+\min \{\lambda, 1-\lambda\} \min \{K, Q\} \\
& -\min \{\mu, 1-\mu\} \min \{K, Q\} \\
\leq & (1-\lambda) g\left(x_{0}\right)+\lambda g\left(x_{1}\right)+\lambda K+(1-\lambda) Q \\
& -\min \{\lambda, 1-\lambda\} \min \{K, Q\}-(1-\lambda) Q \\
& +\min \{\lambda, 1-\lambda\} \min \{K, Q\}-\min \{\mu, 1-\mu\} \min \{K, Q\} \\
\leq & (1-\lambda) g\left(x_{0}\right)+\lambda g\left(x_{1}\right)+\lambda K+(1-\lambda) Q \\
& -\min \{\lambda, 1-\lambda\} \min \{K, Q\},
\end{aligned}
$$

where the first inequality follows from the definition of the function $g$, the second inequality follows from the $(K, Q)$-convexity of the function $f$, the equality holds by rearranging terms, the third inequality follows from the assumption that $f\left(y_{0}\right)-f\left(x_{1}\right) \geq K-Q$, and the last inequality is trivial.

Thus, cases (1)-(4) imply that the function $g$ is $(K, Q)$-convex. Similarly, one can show that $h$ is also $(K, Q)$-convex.

\section{MAIN RESULTS}

In this section, we prove that the cost-to-go function $C_{n}(x)$ is $(K, Q)$-convex and provide a characterization of the optimal policy. Without loss of generality, we assume that $K \geq Q$.

Recall that $H_{n}(x)=L_{n}(x)+\gamma E\left\{C_{n-1}\left(x-\xi_{n}\right)\right\}$. Define

$$
\begin{gathered}
T_{n} \in \operatorname{argmin}_{x}\left\{k x+H_{n}(x)\right\}, \\
t_{n}=\min \left\{x \mid k x+H_{n}(x)=K+k T_{n}+H_{n}\left(T_{n}\right)\right\},
\end{gathered}
$$




$$
\begin{gathered}
t_{n}^{\prime}=\min \left\{x \mid k x+H_{n}(x)=K-Q+k T_{n}+H_{n}\left(T_{n}\right)\right\}, \\
U_{n} \in \operatorname{argmin}_{x}\left\{-q x+H_{n}(x)\right\}, \\
u_{n}=\max \left\{x \mid-q x+H_{n}(x)=Q-q U_{n}+H_{n}\left(U_{n}\right)\right\} .
\end{gathered}
$$

and

$$
u_{n}^{\prime}=\min \left\{x \mid-q x+H_{n}(x)=K-Q-q U_{n}+H_{n}\left(U_{n}\right)\right\} .
$$

In the case where $k+q=0$, we choose $T_{n}=U_{n}$. Later we will see that $T_{n}$ is the order-up-to level, $t_{n}$ is the reorder point, $U_{n}$ is the return-down-to level, $u_{n}$ is the return point, and $t_{n}^{\prime}$ and $u_{n}^{\prime}$ can be used to specify some intervals in which only partial characterization is possible. It is clear that $t_{n} \leq t_{n}^{\prime} \leq T_{n}$ and $u_{n}^{\prime} \leq U_{n} \leq u_{n}$. In addition, we have the following inequalities.

LEMMA 3: $U_{n} \geq T_{n}, u_{n}^{\prime} \geq t_{n}^{\prime}$, and

$$
k\left(U_{n}-T_{n}\right) \geq H_{n}\left(T_{n}\right)-H_{n}\left(U_{n}\right) \geq-q\left(U_{n}-T_{n}\right) .
$$

Proof: By the definition of $T_{n}$ and $U_{n}$, we have that

$$
k T_{n}+H_{n}\left(T_{n}\right) \leq k U_{n}+H_{n}\left(U_{n}\right)
$$

and

$$
-q U_{n}+H_{n}\left(U_{n}\right) \leq-q T_{n}+H_{n}\left(T_{n}\right) .
$$

Inequality (3) implies that $k\left(U_{n}-T_{n}\right) \geq H_{n}\left(T_{n}\right)-H_{n}\left(U_{n}\right)$ and inequality (4) implies that $H_{n}\left(T_{n}\right)-H_{n}\left(U_{n}\right) \geq-q\left(U_{n}-T_{n}\right)$. Adding (3) and (4) together gives that

$$
(k+q)\left(U_{n}-T_{n}\right) \geq 0,
$$

which implies that $U_{n} \geq T_{n}$ when $k+q>0$. By assumption, we have that $T_{n}-U_{n}$ when $k+q=0$.

We now show that $u_{n}^{\prime} \geq t_{n}^{\prime}$. In fact, for any $x<t_{n}^{\prime}$, we have that

$$
\begin{aligned}
-q x+H_{n}(x)= & k x+H_{n}(x)-(k+q) x \\
> & K-Q+k T_{n}+H_{n}\left(T_{n}\right)-(k+q) x \\
= & K-Q-q U_{n}+H_{n}\left(U_{n}\right)+(k+q)\left(T_{n}-x\right)+\left(H_{n}\left(T_{n}\right)-H_{n}\left(U_{n}\right)\right) \\
& +q\left(U_{n}-T_{n}\right) \\
\geq & K-Q-q U_{n}+H_{n}\left(U_{n}\right),
\end{aligned}
$$

where the first inequality follows from the definition of $t_{n}^{\prime}$ and the last inequality follows from the inequality (4) and the assumption that $k+q \geq 0$. Therefore, $u_{n}^{\prime} \geq t_{n}^{\prime}$. 


\section{1. $K \neq Q$}

In this subsection, we assume that $K \neq Q$. We focus on the case that $K>Q \geq 0$. The results for the case $Q>K \geq 0$ follow from a symmetric argument.

TheOREM 3.1: Assume that $K>Q$. The cost-to-go functions $C_{n}(x)$ and $H_{n}(x)$ are $(K, Q)$-convex and it is optimal to set the inventory level $y_{n}(x)$ after a decision is made as follows.

$$
y_{n}(x)= \begin{cases}T_{n} & \text { if } x \leq t_{n} \\ \in\left\{x, T_{n}\right\} & \text { if } x \in\left(t_{n}, t_{n}^{\prime}\right) \\ x & \text { if } x \in\left[t_{n}^{\prime}, u_{n}^{\prime}\right) \\ \in\left[t_{n}^{\prime}, x\right] & \text { if } x \in\left[u_{n}^{\prime}, u_{n}\right) \\ U_{n} & \text { if } x \geq u_{n} .\end{cases}
$$

Proof: By induction. Notice that $C_{0}(x)=0$ and $H_{1}(x)=L_{1}(x)$ are convex and, hence, $(K, Q)$-convex. Now, assume that $C_{n-1}(x)$ is $(K, Q)$-convex; then Lemma 1 (b) and (c) imply that $H_{n}(x)$ is also $(K, Q)$-convex.

Let $O_{n}$ be the set of inventory levels where orders are placed, $R_{n}$ be the set of inventory levels where returns are made, and $E_{n}$ be the set of inventory levels where no transactions are made.

First, notice that

$$
C_{n}(x)=\min \left\{H_{n}(x), \min _{y>x} K+k(y-x)+H_{n}(y), \min _{y>x} Q+q(x-y)+H_{n}(y)\right\} .
$$

In the following we show that (5) characterizes the structure of the optimal policy. We distinguish among five different cases.

Case 1: $x \geq t_{n}^{\prime}$. We claim that it is optimal not to order. In fact, assume that we place an order to raise the inventory level to $y_{n}(x)$. Then

$$
C_{n}(x)=K+k\left(y_{n}(x)-x\right)+H_{n}\left(y_{n}(x)\right) \geq H_{n}(x),
$$

where the inequality follows from the definition of $t_{n}^{\prime}$ and Lemma 1(d)(iv). Thus, staying put at the current inventory level $x$ is no worse than placing an order.

Case 2: $x \leq u_{n}^{\prime}$. We claim that it is optimal not to return. In fact, assume that we reduce the inventory level to $y_{n}(x)$. Then

$$
C_{n}(x)=Q+q\left(x-y_{n}(x)\right)+H_{n}\left(y_{n}(x)\right) \geq H_{n}(x),
$$

where the inequality follows from the definition of $u_{n}^{\prime}$ and Lemma 1(d)(iii) by specializing on the function $-q x+H_{n}(x)$. Thus, staying put at the current inventory level $x$ is no worse than making a return. 
Case 3: $x \leq t_{n}$. The definitions of $t_{n}$ and $T_{n}$ and Case 1 imply that it is optimal to place an order and set $y_{n}(x)=T_{n}$.

Case 4: $x \geq u_{n}$. The definitions of $u_{n}$ and $U_{n}$ and Case 2 imply that it is optimal to make a return and set $y_{n}(x)=U_{n}$.

Case 5: $x \in\left(u_{n}^{\prime}, u_{n}\right)$. We show that the optimal inventory level $y_{n}(x)$ can be chosen so that $y_{n}(x) \in\left[t_{n}^{\prime}, U_{n}\right]$. In fact, for $x \geq U_{n}$, if staying put is not optimal, then from Case 1 it is clearly optimal to make a return to reduce the inventory level to $U_{n}$.

For $x \geq T_{n}$, we claim that the optimal inventory level $y_{n}(x)$ can be chosen so that $y_{n}(x) \geq T_{n}$. Indeed, if $y_{n}(x)<T_{n}$, then we have

$$
\begin{aligned}
C_{n}(x) & =Q+q\left(x-y_{n}(x)\right)+H_{n}\left(y_{n}(x)\right) \\
& =Q+k y_{n}(x)+H_{n}\left(y_{n}(x)\right)+q\left(x-y_{n}(x)\right)-k y_{n}(x) \\
& \geq Q+k T_{n}+H_{n}\left(T_{n}\right)+q\left(x-y_{n}(x)\right)-k y_{n}(x) \\
& =Q+q\left(x-T_{n}\right)+H_{n}\left(T_{n}\right)+(k+q)\left(T_{n}-y_{n}(x)\right) \\
& \geq Q+q\left(x-T_{n}\right)+H_{n}\left(T_{n}\right) \\
& \geq C_{n}(x),
\end{aligned}
$$

where the first inequality follows from the definition of $T_{n}$ and the last inequality follows from the definition of $C_{n}(x)$. Thus, if it is optimal to reduce the inventory to a level below $T_{n}$, then it is also optimal to just reduce the inventory level to $T_{n}$.

Finally, for $x<T_{n}$ and $x \in\left[u_{n}^{\prime}, u_{n}\right]$, we claim that the optimal inventory level $y_{n}(x)$ can be chosen so that $y_{n}(x) \geq t_{n}^{\prime}$. Indeed, if $y_{n}(x)<t_{n}^{\prime}$, then

$$
\begin{aligned}
C_{n}(x) & =Q+q\left(x-y_{n}(x)\right)+H_{n}\left(y_{n}(x)\right) \\
& =Q+k y_{n}(x)+H_{n}\left(y_{n}(x)\right)+q\left(x-y_{n}(x)\right)-k y_{n}(x) \\
& >Q+K-Q+k T_{n}+H_{n}\left(T_{n}\right)+q\left(x-y_{n}(x)\right)-k y_{n}(x) \\
& =K+k\left(T_{n}-x\right)+H_{n}\left(T_{n}\right)+(k+q)\left(x-y_{n}(x)\right) \\
& \geq K+k\left(T_{n}-x\right)+H_{n}\left(T_{n}\right) \\
& \geq C_{n}(x)
\end{aligned}
$$

where the first inequality follows the definition of $t_{n}^{\prime}$ and the last inequality follows from the definition of $C_{n}(x)$. Thus, if it is optimal to reduce the inventory to a level below $t_{n}^{\prime}$, then it is also optimal to increase the inventory level to $T_{n}$.

Cases 1-5 imply the structure of the optimal policy is given by (5).

It remains to prove that the $(K, Q)$-convexity of $H_{n}(x)$ implies the $(K, Q)$ convexity of the function $C_{n}(x)$. In particular, we will prove that for any $x_{0}, x_{1}$, 
and $\lambda \in[0,1]$ with $x_{0} \leq x_{1}$ and $x_{\lambda}=(1-\lambda) x_{0}+\lambda x_{1}$, we have

$$
C_{n}\left(x_{\lambda}\right) \leq(1-\lambda) C_{n}\left(x_{0}\right)+\lambda C_{n}\left(x_{1}\right)+\lambda(K-Q)+\max \{\lambda, 1-\lambda\} Q,
$$

which is exactly the same definition of $(K, Q)$-convexity when $K \geq Q$.

We consider three different cases.

Case 1: $x_{0} \in E_{n}$ or $x_{1} \in E_{n}$. In this case, inequality (6) follows from Lemma 2.

Case 2: $x_{0}, x_{1} \in O_{n}$ or $x_{0}, x_{1} \in R_{n}$. In this case, inequality (6) follows from Lemma 2.

Case 3: $\quad x_{0} \in O_{n} \quad$ and $\quad x_{1} \in R_{n}$. Then $C_{n}\left(x_{0}\right)=K+k\left(T_{n}-x_{0}\right)+H_{n}\left(T_{n}\right)$ and $C_{n}\left(x_{1}\right)=Q+q\left(x_{1}-y_{1}\right)+H_{n}\left(y_{1}\right)$, for some $y_{1}<x_{1}$. Notice that from our above analysis, if $x_{1} \geq T_{n}$, then $y_{1} \geq T_{n}$. We distinguish between three cases:

Subcase 1: $\quad x_{\lambda} \leq T_{n}$.

$$
\begin{aligned}
C_{n}\left(x_{\lambda}\right) \leq & K+k\left(T_{n}-x_{\lambda}\right)+H_{n}\left(T_{n}\right) \\
= & (1-\lambda) C_{n}\left(x_{0}\right)+\lambda C_{n}\left(x_{1}\right) \\
& +\lambda\left(\left(K+k\left(T_{n}-x_{1}\right)+H_{n}\left(T_{n}\right)\right)-\left(Q+q\left(x_{1}-y_{1}\right)+H_{n}\left(y_{1}\right)\right)\right) \\
= & (1-\lambda) C_{n}\left(x_{0}\right)+\lambda C_{n}\left(x_{1}\right) \\
& +\lambda\left(K-Q-(k+q)\left(x_{1}-y_{1}\right)+\left(k T_{n}+H_{n}\left(T_{n}\right)\right)-\left(k y_{1}+H_{n}\left(y_{1}\right)\right)\right) \\
\leq & (1-\lambda) C_{n}\left(x_{0}\right)+\lambda C_{n}\left(x_{1}\right)+\lambda(K-Q),
\end{aligned}
$$

where the last inequality holds since $k+q \geq 0$ and $T_{n}$ is the global minimizer of function $k x+H_{n}(x)$.

Subcase 2: $\quad x_{\lambda} \geq y_{1} \geq T_{n}$.

$$
\begin{aligned}
C_{n}\left(x_{\lambda}\right) \leq & Q+q\left(x_{\lambda}-y_{1}\right)+H_{n}\left(y_{1}\right) \\
= & (1-\lambda) C_{n}\left(x_{0}\right)+\lambda C_{n}\left(x_{1}\right) \\
& +(1-\lambda)\left(\left(Q+q\left(x_{0}-y_{1}\right)+H_{n}\left(y_{1}\right)\right)-\left(K+k\left(T_{n}-x_{0}\right)+H_{n}\left(T_{n}\right)\right)\right) \\
= & (1-\lambda) C_{n}\left(x_{0}\right)+\lambda C_{n}\left(x_{1}\right)+(1-\lambda)\left(Q-K-(k+q)\left(T_{n}-x_{0}\right)\right. \\
& \left.+\left(-q y_{1}+H_{n}\left(y_{1}\right)\right)-\left(-q T_{n}+H_{n}\left(T_{n}\right)\right)\right) \\
\leq & (1-\lambda) C_{n}\left(x_{0}\right)+\lambda C_{n}\left(x_{1}\right)+(1-\lambda)(Q-K),
\end{aligned}
$$

where the second inequality holds since $-q y_{1}+H_{n}\left(y_{1}\right) \leq-q T_{n}+H_{n}\left(T_{n}\right)$. 
Subcase 3: $\quad T_{n} \leq x_{\lambda} \leq y_{1}$. Let $x_{\lambda}=(1-\mu) T_{n}+\mu y_{1}$. Then we have that

$$
\left.\mu\left(y_{1}-T_{n}\right)=\lambda\left(x_{1}-x_{0}\right)-T_{n}-x_{0}\right)
$$

and

$$
\begin{aligned}
C_{n}\left(x_{\lambda}\right) \leq & H_{n}\left(x_{\lambda}\right) \\
\leq & (1-\mu) H_{n}\left(T_{n}\right)+\mu H_{n}\left(y_{1}\right)+\mu(K-Q)+\max \{\mu, 1-\mu\} Q \\
= & (1-\lambda) C_{n}\left(x_{0}\right)+\lambda C_{n}\left(x_{1}\right)+\lambda(K-Q)+(\mu-\lambda)(K-Q) \\
& +\max \{\mu, 1-\mu\} Q+(\mu-\lambda)\left(H_{n}\left(y_{1}\right)-H_{n}\left(T_{n}\right)\right) \\
& -(1-\lambda)\left(K+k\left(T_{n}-x_{0}\right)\right)-\lambda\left(Q+q\left(x_{1}-y_{1}\right)\right) \\
= & (1-\lambda) C_{n}\left(x_{0}\right)+\lambda C_{n}\left(x_{1}\right)+\lambda(K-Q) \\
& +(\mu-\lambda)(K-Q)+\max \{\mu, 1-\mu\} Q-(1-\lambda) K-\lambda Q+\Delta \\
= & (1-\lambda) C_{n}\left(x_{0}\right)+\lambda C_{n}\left(x_{1}\right)+\lambda(K-Q) \\
& +\max \{\mu, 1-\mu\} Q-\mu Q-(1-\mu) K+\Delta \\
\leq & (1-\lambda) C_{n}\left(x_{0}\right)+\lambda C_{n}\left(x_{1}\right)+\lambda(K-Q)+\Delta,
\end{aligned}
$$

where

$$
\Delta=(\mu-\lambda)\left(H_{n}\left(y_{1}\right)-H_{n}\left(T_{n}\right)\right)-(1-\lambda) k\left(T_{n}-x_{0}\right)-\lambda q\left(x_{1}-y_{1}\right)
$$

and the second inequality follows from the $(K, Q)$-convexity of function $H_{n}$. Thus, it suffices to prove that $\Delta \leq 0$. We distinguish between two cases.

First, if $\mu \geq \lambda$, then

$$
\begin{aligned}
\Delta & \leq(\mu-\lambda) q\left(y_{1}-T_{n}\right)-(1-\lambda) k\left(T_{n}-x_{0}\right)-\lambda q\left(x_{1}-y_{1}\right) \\
& =q \lambda\left(x_{1}-x_{0}\right)-q\left(T_{n}-x_{0}\right)-\lambda q\left(y_{1}-T_{n}\right)-(1-\lambda) k\left(T_{n}-x_{0}\right)-\lambda q\left(x_{1}-y_{1}\right) \\
& =-(1-\lambda)(k+q)\left(T_{n}-x_{0}\right) \\
& \leq 0,
\end{aligned}
$$

where the first inequality holds since $-q y_{1}+H_{n}\left(y_{1}\right) \leq-q T_{n}+H_{n}\left(T_{n}\right)$, the first equality follows from (7), and the last equality follows from simple algebra.

If $\mu \leq \lambda$, then

$$
\begin{aligned}
\Delta & \leq(-\mu+\lambda) k\left(y_{1}-T_{n}\right)-(1-\lambda) k\left(T_{n}-x_{0}\right)-\lambda q\left(x_{1}-y_{1}\right) \\
& =-k \lambda\left(x_{1}-x_{0}\right)+k\left(T_{n}-x_{0}\right)+\lambda k\left(y_{1}-T_{n}\right)-(1-\lambda) k\left(T_{n}-x_{0}\right)-\lambda q\left(x_{1}-y_{1}\right) \\
& =-\lambda(k+q)\left(x_{1}-y_{1}\right) \\
& \leq 0,
\end{aligned}
$$

where the first inequality holds since $H_{n}\left(y_{1}\right)-H_{n}\left(T_{n}\right) \geq-k\left(y_{1}-T_{n}\right)$, the first equality follows from (7), and the last equality follows from simple algebra. 
From the above proof, we know that when the initial inventory level $x$ is no more than the reorder point $t_{n}$, an order is placed to raise the inventory level to $T_{n}$. On the other hand, when the initial inventory level $x$ is no less than the return point $u_{n}$, we decrease the inventory level to the return-down-to level $U_{n}$. For inventory levels between $\left(t_{n}^{\prime}, u_{n}^{\prime}\right)$, no action is taken. However, for inventory levels in other intervals, only partial characterization is available. For instance, $y_{n}(x) \in\left\{x, U_{n}\right\}$ for $x \in\left[U_{n}, u_{n}\right]$ and $y_{n}(x) \geq T_{n}$ for $x \geq T_{n}$. Also observe that Lemma 1(d)(v) implies that $t_{n}^{\prime}$ in Theorem 3.1 can be replaced by $\min \left(t_{n}^{\prime},\left(t_{n}+T_{n}\right) / 2\right)$.

It is interesting to notice that the optimal policy characterized by Theorem 3.1 is similar to the optimal policy identified by Neave. The main difference is that when $x \in\left[t_{n}^{\prime}, u_{n}^{\prime}\right)$ the optimal policy identified by Theorem 3.1 specifies that no action is needed, whereas in Neave's policy, it is not clear whether a decision need to be made.

\section{2. $K=Q$}

In this subsection, we focus on a special case of the stochastic cash balance problem where $K=Q>0$.

Theorem 3.2: Assume that $K=Q$. The cost-to-go functions $C_{n}(x)$ and $H_{n}(x)$ are symmetric $K$-convex and the optimal inventory level $y_{n}(x)$ after a decision is made satisfies

$$
y_{n}(x)= \begin{cases}T_{n} & \text { if } x \leq t_{n} \\ \in\left\{x, T_{n}\right\} & \text { if } x \in\left(t_{n},\left(t_{n}+T_{n}\right) / 2\right) \\ x & \text { if } x \in\left[\left(t_{n}+T_{n}\right) / 2,\left(U_{n}+u_{n}\right) / 2\right] \\ \in\left\{x, U_{n}\right\} & \text { if } \left.x \in\left[U_{n}+, u_{n}\right) / 2, u_{n}\right) \\ U_{n} & \text { if } x \geq u_{n} .\end{cases}
$$

Proof: The symmetric $K$-convexity of functions $C_{n}(x)$ and $H_{n}(x)$ follows from Theorem 3.1 since the $(K, Q)$-convexity reduces to the symmetric $K$-convexity when $K=Q$. It remains to characterize the optimal inventory policy [i.e., to show that the structure of $y_{n}(x)$ is as defined in (8)]. In fact, from the definitions of $t_{n}, T_{n}, u_{n}$, and $U_{n}$ and Lemma 1(d)(v), one can see that no order is placed when $x \geq\left(t_{n}+T_{n}\right) / 2$ since $k x+H_{n}(x)$ is symmetric $K$-convex. Similarly, no return is placed when $x \leq$ $\left(U_{n}+u_{n}\right) / 2$ since $-q x+H_{n}(x)$ is symmetric $K$-convex. Therefore, the structure of the optimal policy follows from Theorem 3.1.

Notice that when $x \in\left(t_{n},\left(t_{n}+T_{n}\right) / 2\right)$, the optimal policy is either do nothing or increase inventory to $T_{n}$. Similarly, when $\left.x \in\left(U_{n}+u_{n}\right) / 2, u_{n}\right)$, the optimal policy is either do nothing or reduce inventory to $U_{n}$. Thus, $T_{n}$ and $U_{n}$ are the unique order-up-to level and return-down-to level, respectively.

It is also appropriate to point out that Neave [9] provided an example that demonstrates that even when $L_{n}$ is symmetric and convex, $k=q=0, K=Q$, and the distribution function of the demand is symmetric, it is possible to order for 
$\left.x \in\left(t_{n},+T_{n}\right) / 2\right)$ or to return for $\left.x \in\left(U_{n}+u_{n}\right) / 2, u_{n}\right)$ [i.e., the sets $O_{n} \cap\left(t_{n},\left(t_{n}+\right.\right.$ $\left.\left.T_{n}\right) / 2\right)$ and $R_{n} \cap\left(U_{n},\left(U_{n}+u_{n}\right) / 2\right)$ might not be empty]. Furthermore, in his example, the functions $C_{n}(x)$ and $H_{n}(x)$ are not $K$-convex.

\section{CONCLUDING REMARKS}

In this article, we show that new concepts such as symmetric $K$-convexity and $(K, Q)$ convexity, can be very useful for the stochastic cash balance problem. It is easy to see that these concepts can be applied to extend our results to the stochastic cash balance problem with time-dependent parameters.

It is appropriate to point out that similar structural results of the optimal policy for the stochastic cash balance problem have been derived by Neave [9], although, as observed earlier, his analysis is not complete. He constructed by induction a convex function $\underline{C}_{n}(x)$ such that $\underline{C}_{n}(x) \leq \underline{C}_{n}(x) \leq \underline{C}_{n}(x)+\max \{K, Q\}$ for any $x$. The lower and upper bounds of the function $\underline{C}_{n}(x)$ allows Neave to characterize the structure of the optimal policy. Interestingly, the symmetric $K$-convexity approach used in our article implies the existence of those lower and upper bounds for $C_{n}(x)$ with similar properties. Indeed, we have the following result, whose proof is presented in the Appendix.

THeOREM 4.1: Assume that $f: \Re \rightarrow \Re$ is $(K, Q)$-convex. There exists a convex function $\underline{f}(x)$ such that

$$
\underline{f}(x) \leq f(x) \leq \underline{f}(x)+\max \{K, Q\} \text { for any } x .
$$

\section{Acknowledgments}

This research was supported in part by the Center of eBusiness at MIT, ONR Contracts N00014-95-1-0232 and N00014-01-1-0146, and by NSF Contracts DMI-9732795, DMI-0085683 and DMI-0245352. This research is also supported by National Science Foundation Grant CCR-9731273, CMMI-0653909, and CMMI-0926845 ARRA.

We thank one anonymous referee for valuable suggestions to improve the paper.

Notes

1. If $l_{n}(z)=O\left(|z|^{\rho}\right)$ for some integer $\rho$, it suffices to assume that $E\left\{\left|\xi_{n}\right|^{\rho}\right\}<\infty$.

2. Note that the $S$ and $s^{\prime}$ for the function $g(x)=-q x+H_{n}(x)$ are different from the $S$ and $s^{\prime}$ defined for the function $g(x)=k x+H_{n}(x)$.

3. This result first appeared in the earlier version (2003) of this article and also appeared in SimchiLevi, Chen, and Bramel [11] as an exercise. We note that in the published version of Ye and Duenyas [13], referred to as Ye and Duenyas [14], they present an extension of this result, in which the domain of the function $f$ can be an interval.

\section{References}

1. Chen, X. (2003). Coordinating inventory control and pricing strategies with random demand and fixed ordering cost. Ph.D. dissertation, Massechusetts Institute of Technology, Cambridge, MA. 
2. Chen, X. \& Simchi-Levi, D. (2004). Coordinating inventory control and pricing strategies with random demand and fixed ordering cost: The finite horizon Case. Operations Research 29: 698-723.

3. Chen, X. \& Simchi-Levi, D. (2004). Coordinating inventory control and pricing strategies with random demand and fixed ordering cost: The infinite horizon case. Mathematics of Operations Research 52: 887-896.

4. Eppen, G.D. \& Fama, E.F. (1999). Cash balance and simple dynamic portofolio problems with proportional costs. International Econmomics Review 10: 119-133.

5. Feinberg, E. \& Lewis, M. (2005). Optimality of four-threshold policies inventory systems with customer returns and borrowing/storage options. Probability in the Engineering and Informational Sciences 19(1):45-71.

6. Feinberg, E. \& Lewis, M. (2007). Optimality inequalities for average cost markov decision processes and the stochastic cash balance problem. Mathematics of Operations Research 32(4): 769-783.

7. Fleischmann, M., Bloemhof-Ruwaarda, J., Dekker, R., van der Laana, E., van Nunena, J., \& Van Wassenhove, L. (1997). Quantitative models for reverse logistics: A review. European Journal on Operational Research 103(1): 1-47.

8. Girgis, N.M.(1968). Optimal cash balance levels. Management Science 15: 130-140.

9. Neave, E.H. (1970). The stochastic cash balance problem with fixed costs for increases and decreases. Management Science 16: 472-490.

10. Scarf, H. (1960). The optimality of $(s, S)$ policies for the dynamic inventory problem. In Arrow, K., Karlin, S. \& Suppes, P. (eds.), Proceedings of the 1st Stanford symposium on mathematical methods in the social sciences. Stanford University Press, Stanford, CA.

11. Simchi-Levi, D., Chen, X., \& Bramel, J. (2005). The logic of logistics: Theory, algorithms, and applications for logistics and supply chain management, 2nd ed. New York: Springer-Verlag.

12. Whisler, W.D. (1967). A stochastic inventory model for rented equipment. Management Science 13: 640-647.

13. Ye, Q. \& Duenyas, I. (2003). Optimal joint capacity investment and pricing/production quantity decisions with random and fixed capacity adjustment costs. Working paper, University of Michigan.

14. Ye, Q. \& Duenyas, I. (2007). Optimal capacity investment decisions with two-sided fixed-capacity adjustment costs. Operations Research 55(2): 272-283.

\section{APPENDIX}

Proof of Theorem 4.1: Assume without loss of generality that $K \geq Q$. Since a $(K, Q)$-convex function is also symmetric $K$-convex, it suffices to prove the result for a symmetric $K$-convex function $f$.

Let $\underline{f}(x)$ be the lower convex envelope of function $f(x)$; that is,

$$
\underline{f}(x)=\inf \left\{(1-\lambda) f\left(x_{0}\right)+\lambda f\left(x_{1}\right) \mid x=(1-\lambda) x_{0}+\lambda x_{1}, \lambda \in[0,1]\right\} .
$$

Obviously, $f(x)$ is a lower bound of $f(x)$. In addition, for a given $x$, any $\lambda \in[0,1]$ and $x_{0}$ and $x_{1}$ with $x=(1-\lambda) x_{0}+\lambda x_{1}$, we have from the definition of symmetric $K$-convexity that

$$
f(x) \leq(1-\lambda) f\left(x_{0}\right)+\lambda f\left(x_{1}\right)+\max \{1-\lambda, \lambda\} K \leq(1-\lambda) f\left(x_{0}\right)+\lambda f\left(x_{1}\right)+K .
$$

Hence, $f(x) \leq f(x)+K$.

We now show that $f(x)$ is convex. Notice that $f(x)$ is convex if and only if the epigraph of $f(x)$, epi $(\underline{f})=\{(x, w) \overline{\mid} \underline{f}(x) \leq w\}$, is convex. However, it is not difficult to see that epi $(\underline{f})$ is the convexification of epi $(f)$ and, hence, is a convex set. Thus, $f(x)$ is convex. 